\title{
Polarization control of high transmission/reflection switching by all-dielectric metasurfaces
}

Toshihiko Shibanuma, Stefan A. Maier, and Pablo Albella

Citation: Appl. Phys. Lett. 112, 063103 (2018); doi: 10.1063/1.5018783

View online: https://doi.org/10.1063/1.5018783

View Table of Contents: http://aip.scitation.org/toc/apl/112/6

Published by the American Institute of Physics

\section{Articles you may be interested in}

Near-field plasmonic beam engineering with complex amplitude modulation based on metasurface

Applied Physics Letters 112, 073104 (2018); 10.1063/1.5013327

An optically transparent metasurface for broadband microwave antireflection

Applied Physics Letters 112, 073504 (2018); 10.1063/1.5018017

Electrically tunable all-dielectric optical metasurfaces based on liquid crystals

Applied Physics Letters 110, 071109 (2017); 10.1063/1.4976504

Invited Article: Broadband highly efficient dielectric metadevices for polarization control

APL Photonics 1, 030801 (2016); 10.1063/1.4949007

High-efficiency and low-loss gallium nitride dielectric metasurfaces for nanophotonics at visible wavelengths Applied Physics Letters 111, 221101 (2017); 10.1063/1.5007007

Active tuning of high- $Q$ dielectric metasurfaces

Applied Physics Letters 111, 053102 (2017); 10.1063/1.4997301

\section{Conference Proceedings}




\title{
Polarization control of high transmission/reflection switching by all-dielectric metasurfaces
}

\author{
Toshihiko Shibanuma, ${ }^{1,2}$ Stefan A. Maier, ${ }^{1,3, a)}$ and Pablo Albella ${ }^{1,4, b)}$ \\ ${ }^{1}$ The Blackett Laboratory, Department of Physics, Imperial College London, London SW7 2AZ, \\ United Kingdom \\ ${ }^{2}$ Central Technical Research Laboratory, JXTG Nippon Oil \& Energy Corporation, 8, Chidori-cho, Naka-ku, \\ Yokohama 231-0815, Japan \\ ${ }^{3}$ Chair in Hybrid Nanosystems Faculty of Physics, Ludwig-Maximilians-Universität München, \\ 80799 München, Germany \\ ${ }^{4}$ University Institute for Intelligent Systems and Numerical Applications in Engineering (SIANI), \\ University of Las Palmas de Gran Canaria, 35017 Las Palmas de Gran Canaria, Spain
}

(Received 9 December 2017; accepted 24 January 2018; published online 7 February 2018)

\begin{abstract}
Metasurfaces built of high refractive dielectric nanostructures could play a key role in controlling the electromagnetic wave propagation, due to their low energy losses and their ability to excite not only electric but also magnetic resonances. In this study, we theoretically and experimentally demonstrate that an array of high-index dielectric nanodimers can perform as tuneable metasurfaces that can be switched from a high transmitter to a high reflector, by just changing the linear polarization of excitation. The incident polarization alters the hybridization mode of the excited electric and magnetic dipoles in the dimer, and this leads to either spectral overlap or separation of the two dipoles. The hybridization of the electric and magnetic modes modifies the effective permittivity and permeability of the tuneable dielectric metasurface, exhibiting the high transmission and reflection that can be easily switched by simply changing the linear polarization. Published by AIP Publishing. https://doi.org/10.1063/1.5018783
\end{abstract}

Metasurfaces are flat, ultrathin optical components artificially consisting of arrays of subwavelength optical nanoantennas. ${ }^{1-6}$ The optical effects that metasurfaces show can be mainly determined by the resonant properties of the constituting nanostructures. Plasmonic nanoantennas made of noble metals have been commonly employed as nanoresonators forming the unit cells of metasurfaces, due to their ability to confine and control the light even below the diffraction limit. ${ }^{7,8}$ However, ohmic losses and consequently undesirable heat are inevitable when using plasmonic nanoparticles especially in the visible and near infrared (NIR) regime. This has hampered plasmonic metasurfaces from being applied in real applications. ${ }^{9}$

Recently, metasurfaces built of high refractive index dielectric nanoantennas have emerged as alternatives for flat optical components. ${ }^{10-16}$ Nanoparticles made of high-index dielectrics, such as $\mathrm{Si}, \mathrm{GaAs}$, and $\mathrm{GaP}$, can exhibit strong scattering and near field enhancement by exciting Mie resonances without substantial energy losses. ${ }^{17-22}$ Moreover, magnetic resonances can be easily excited in dielectric nanoantennas together with the electric ones. ${ }^{18,19,23}$ These features of high-index dielectric nanoparticles enable metasurfaces to offer unique optical effects. One example is the high resonant transmission efficiency from a metasurface using low aspectratio dielectric nanoantennas as Huygens' sources, showing a spectral overlap between the electric and magnetic dipolar resonances. ${ }^{24-27}$ On the other hand, perfect flat reflectors have also been reported using metasurfaces built of high

\footnotetext{
${ }^{\text {a)} E-m a i l: ~ p a b l o . a l b e l l a @ u l p g c . e s ~}$

${ }^{\text {b) }}$ E-mail: s.maier@imperial.ac.uk
}

aspect-ratio dielectric resonators, by achieving the spectral separation of the two resonances. ${ }^{28-31}$

However, the tuneable control over the switching of high transmission/reflection has been barely explored. ${ }^{32,33}$ Very recently, a circular dichroism waveplate was proposed using a Si-based all-dielectric chiral metasurface, showing high transmittance or reflectance depending on the circular polarization. ${ }^{34}$ The switching from transmissive to reflective properties by incident linear polarization was reported using metallic metamaterials; however, the efficiency is still low in the visible and NIR regime because of ohmic losses of metals. ${ }^{35}$

In this letter, we theoretically and experimentally demonstrate how high-index dielectric metasurfaces when conveniently designed can act as a switch from high transmission to high reflection by simply changing the linear polarization state of light in the NIR region. These metasurfaces are composed of an array of Si dimeric nanoparticles, in which the excited electric and magnetic dipoles are coupled and form hybridized modes. ${ }^{36-38}$ The energy level of the hybridized modes can be controlled by the incident polarization, allowing these metasurfaces to have anisotropic optical responses. The working principle is theoretically explored with numerical simulations using an array of Si spherical nanodimers in air. For the experimental demonstration, we measure the transmission and reflection of an array of Si disk nanodimers that are fabricated by electron beam lithography on a sapphire substrate. These ultrathin metasurfaces can work either as transmitters or as reflectors by simply controlling the linear polarization of the incident light, showing that they could be one of the key components for tuneable optical flat devices.

First, we explore the required conditions to achieve either high transmission or high reflection from the metasurface 
built of arrays of nanoresonators. For simplicity, we consider the transmission $(T)$ and reflection $(R)$ of a plane wave illuminating at normal incidence the interface between air and a semi-infinite medium, which are defined as follows:

$$
\begin{gathered}
R=\frac{\left(Z^{\prime}-1\right)^{2}+Z^{\prime \prime 2}}{\left(Z^{\prime}+1\right)^{2}+Z^{\prime \prime 2}}, \\
T=1-R=\frac{4 Z^{\prime}}{\left(Z^{\prime}+1\right)^{2}+Z^{\prime \prime 2}},
\end{gathered}
$$

where $Z=Z^{\prime}+i Z^{\prime \prime}$ is the complex impedance of the metasurface calculated by $Z=\sqrt{\mu / \varepsilon}$, in which $\varepsilon\left(=\varepsilon^{\prime}+i \varepsilon^{\prime \prime}\right)$ is the complex permittivity and $\mu\left(=\mu^{\prime}+i \mu^{\prime \prime}\right)$ is the complex permeability.

First, the near unity transmission, which requires $R=0$, can be achieved when $Z^{\prime}=1$ and $Z^{\prime \prime}=0$. Simple arithmetic manipulation gives the requirements of $\varepsilon$ and $\mu$

$$
\begin{gathered}
\varepsilon^{\prime}=\mu^{\prime}, \\
\varepsilon^{\prime \prime}=\mu^{\prime \prime}=0 .
\end{gathered}
$$

These two conditions can be satisfied in a high-index dielectric metasurface using the electric and magnetic resonances of its constituent nanoantennas, by tuning them so that they show a perfect spectral overlap. ${ }^{25}$ The effective permittivity and permeability of a metasurface can be equal when the Mie coefficients $a_{1}$ and $b_{1}$ of component nanoantennas are equal. ${ }^{39}$ This can be fulfilled in high-index dielectric nanoantennas by analogy with the first Kerker condition, which provides unidirectional forward scattering when $a_{1}=b_{1}$. The first Kerker condition has been demonstrated using highindex dielectric nanoantennas recently. ${ }^{40-42}$ Furthermore, the electric and magnetic resonances can be achieved with small absorption by using high-index dielectric nanoantennas, leading to the satisfaction of Eq. (4). ${ }^{29}$ In fact, the near unity transmission with full $2 \pi$ phase control has been demonstrated using metasurfaces made of dielectric nanodisks with a low aspect ratio. ${ }^{13,25-27}$

Second, the near unity reflection, which is $R=1$, is explored. In this case, the required condition is that $Z^{\prime}=0$ in Eq. (2) or in terms of $\varepsilon$ and $\mu$ that

$$
\begin{gathered}
\frac{\varepsilon^{\prime}}{\mu^{\prime}}<0, \\
\varepsilon^{\prime \prime} \mu^{\prime}-\varepsilon^{\prime} \mu^{\prime \prime}=0 .
\end{gathered}
$$

High-index dielectric metasurfaces again can fulfill these two conditions. When the electric and magnetic dipolar resonances of the high-index dielectric nanoantennas forming the metasurfaces are separated sufficiently, dispersion-like resonances occur in the real part of effective permittivity and permeability at different wavelengths. This provides a certain wavelength range where either $\varepsilon^{\prime}$ or $\mu^{\prime}$ can be negative, satisfying Eq. (5). ${ }^{28-30,43,44}$ Although Eq. (6) looks more restrictive, low loss dielectric metasurfaces have near zero $\varepsilon^{\prime \prime}$ or $\mu^{\prime \prime}$, thus allowing its fulfilment. In fact, perfect reflection has already been theoretically and experimentally demonstrated using dielectric metasurfaces designed with high aspect ratio dielectric nanoantennas. ${ }^{29,30}$
To attain a switch between high transmission and reflection of dielectric metasurfaces without changing their geometries, we propose the use of mode hybridization in arrays of high-index dielectric dimers. Similar to the well-known interactions of the electric dipoles of plasmonic nanoparticles, ${ }^{45-47}$ electric and magnetic resonances excited in the dielectric nanoparticles can also show interaction between the resonances, and hence, mode hybridizations can occur. Here, only hybridization modes of electric-electric and magnetic-magnetic dipole interactions are considered. The higher-order modes, such as quadrupolar excitations, in general, concentrate the electric field enhancement inside the particles, leading to less strong interactions. ${ }^{36}$ Also, heterogeneous electric-magnetic dipole interactions can be present in dimers of high-index dielectric nanoparticles; however, the contribution of this coupling is much smaller than the homogeneous electric-electric or magnetic-magnetic coupling, and it can be neglected, as previously reported. $^{36,38}$

Figures 1(a) and 1(b) show the schematic images of the electric and magnetic dipoles excited in a high-index dielectric dimer under the illumination of light polarized along (TM polarization) and perpendicular to (TE polarization) the dimer axis. ${ }^{38}$ The TM polarized incidence excites electric dipoles along the dimer axis, inducing the bonding electricelectric dipole interaction, similar to the plasmonic dimer case. ${ }^{45}$ This interaction can lower the energy of the excited electric dipoles, resulting in a redshift of the resonant wavelength of the electric mode, as shown in Fig. 1(c). At the same time, the magnetic dipoles are excited in each particle
TM

a

c
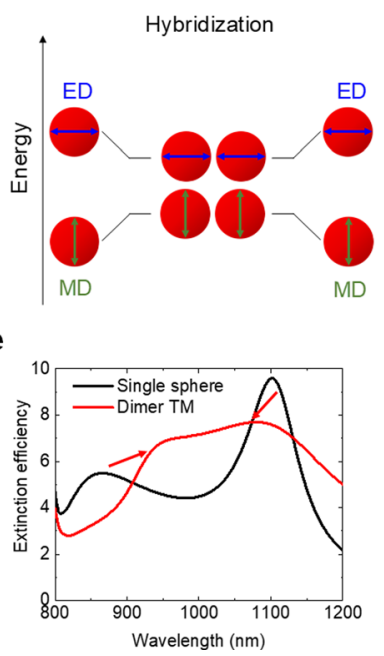

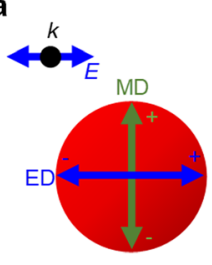

TE

b

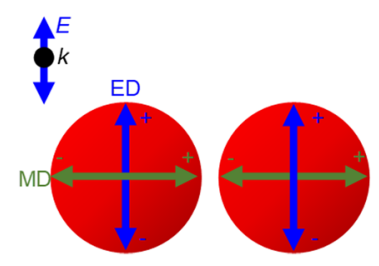

d

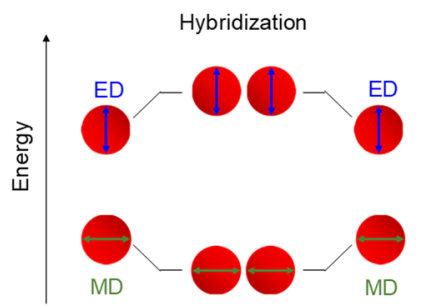

f

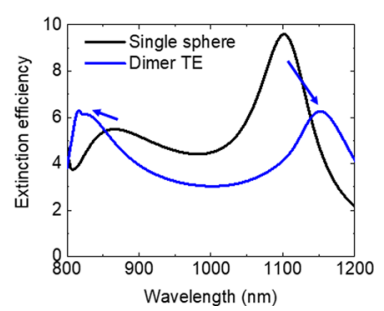

FIG. 1. Polarization dependent dipole excitation and hybridization of the $\mathrm{Si}$ dimer illuminated by [(a), (c), and (e)] TM polarization and [(b), (d), and (f)] TE polarization. (a) and (b) Schematic images of excited electric and magnetic dipoles. (c) and (d) Mode hybridization of the Si dimer. (e) and (f) Extinction efficiency spectra. 
but perpendicular to the dimer axis. The repulsive force between the poles in the particles increases the energy level of the magnetic mode, leading to a blue-shift of the resonant wavelength.

On the other hand, the other polarization (TE) results in an opposite effect, both on the spectral shift and on the energy diagram of the dielectric dimer system. As shown in Fig. 1(b), the magnetic dipoles are excited along the dimer axis and form the bonding modes to red shift its energy level by the TE polarized light [Fig. 1(d)]. The electric modes, in contrast, are blue-shifted by the coupling of the electric dipoles excited perpendicular to the dimer axis. Thus, the optical response and hybridization of modes in a high-index dielectric dimer can be controlled by changing the linear polarization state of the incident light.

To confirm the polarization dependent mode hybridizations described before, we numerically investigate isolated dimers of spherical Si nanoparticles in air. After optimization, we choose a sphere diameter of $300 \mathrm{~nm}$ and a gap distance of $10 \mathrm{~nm}$ to be the unit cell of the metasurface. Figures 1(e) and 1(f) show a comparison of the calculated extinction spectra of the Si spherical dimer and the one of the just isolated Si nanospheres that form the dimer. These spectra are numerically calculated by the finite-difference time-domain (FDTD) method under excitation with linearly polarized normal incidence. The isolated spherical nanoparticle showed the electric and magnetic dipolar resonances at $\lambda=860 \mathrm{~nm}$ and $\lambda=1110 \mathrm{~nm}$, respectively. When the Si dimer was excited by the TM polarized light, the resonances of electric and magnetic dipoles were red-shifted and blue-shifted, respectively. As a result, the two resonances approached each other and showed a spectral overlap around $\lambda=1000 \mathrm{~nm}$. The TE polarized light, on the other hand, blue-shifted and redshifted the electric and magnetic dipolar resonances, respectively, causing the large spectral separation between the two resonant wavelengths. These results agreed well with the model of mode hybridization, which presents the energy shift depending on the incident polarization. ${ }^{38}$ Thus, the illumination of TM and TE polarized incident light can give rise to the spectrum overlap and separation in the extinction spectrum of the Si nanodimer, respectively.

As discussed above by using Eqs. (1)-(6), high transmission and reflection of the metasurfaces can be achieved by simple manipulation of their effective permittivity and permeability, ${ }^{39,43,44}$ which can be tuned by the electric and magnetic resonances of the constituent dielectric nanoparticles. $^{25,28}$ An array of high-index dielectric dimers showing the pursued spectral overlap or separation between the electric and magnetic dipolar resonances depending on the incident polarization could work as a metasurface which can switch the high transmission and reflection simply by changing the linear polarization of the incident light.

We numerically simulated the array of the Si spherical dimers using the FDTD method with an incident plane wave normal to the dimer array [Fig. 2(a)]. Figure 2(b) shows the transmission and reflection of the dimer array having the dimensions described above (diameter of $300 \mathrm{~nm}$ and separation distance of $10 \mathrm{~nm}$ ). The periodicities of the array along (x) and perpendicular (y) to the dimer axis were set to $790 \mathrm{~nm}$ and $480 \mathrm{~nm}$, respectively. The light illumination of a

b
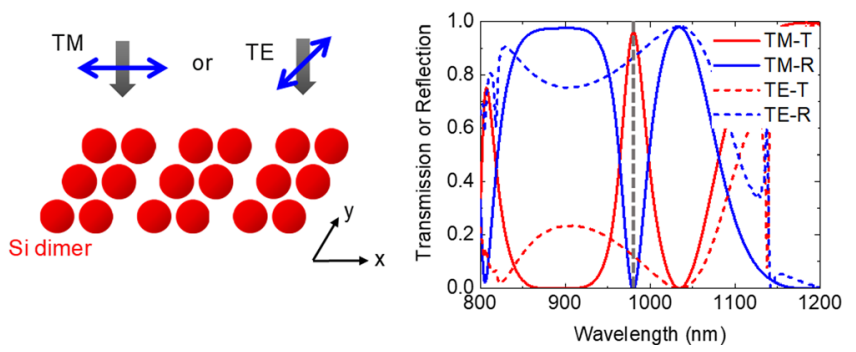

FIG. 2. (a) Schematic image of the Si dimer array in air illuminated by the linearly polarized incident light. (b) Transmission (red lines) and reflection (blue lines) spectra of the Si dimer array under the illumination of TM (solid lines) and TE (broken lines) polarization.

TM polarization evoked a high transmission of $96.5 \%$ at $\lambda=975 \mathrm{~nm}$. However, when the dimer array was excited by the TE polarized light, the transmission peak disappeared and the reflection remained as high as $\mathrm{R}=86 \%$ at $\lambda=975 \mathrm{~nm}$. The switching wavelength between high transmission and reflection by the incident polarization agreed well with the wavelength where the spectral overlap and separation were observed in the extinction spectrum of the Si spherical dimer unit [Figs. 1(e) and 1(f)]. Thus, the high transmission to reflection switching was theoretically demonstrated using the linear polarization tuning of the electric and magnetic hybridizations in a high-index dielectric dimer. Compared to a conventional linear polarizer, such as a wire grid polarizer, one of the advantages that we can obtain from the metasurface of dielectric dimers is the selectivity in wavelength. The transmission at TE-polarization exhibited a clear narrow peak, showing potential to be applied for sensing applications. Moreover, the resonant wavelengths are scalable simply by changing the dimensions of the unit nanodisk. Note that the reflection observed from the array of $\mathrm{Si}$ spherical dimers under the excitation of TE polarized light was a bit lower than the reflection obtained using Si cylindrical nanoparticles with a high aspect ratio. ${ }^{29}$ This might be because the spectral separation of the electric and magnetic modes in the Si dimer in the case of TE-polarization was too large to achieve the high reflection. The center wavelength of the large spectral separation is far from both of the resonances, and hence, the absolute values of effective permittivity and permeability are small, leading to the small imaginary part of the refractive index and allowing the evanescent tunneling to occur. This can cause the reduction in the reflection value of the metasurface. ${ }^{28,29}$

To experimentally demonstrate the switch between high transmission and reflection of a high-index dielectric metasurface, we fabricated an array of Si disk nanodimers on a sapphire substrate using electron beam lithography and reactive ion etching. Positive tone resist, PMMA [poly(methyl methacrylate)], was spin-coated onto a Si-on-sapphire substrate and baked at $180^{\circ} \mathrm{C}$ for $5 \mathrm{~min}$. The substrate was exposed with an electron beam followed by a development procedure in a MIBK (methyl isobutyl ketone):IPA (isopropanol) =1:3 solvent. The nanostructured PMMA was covered with a $40 \mathrm{~nm} \mathrm{Cr}$ layer deposited by thermal evaporation and then removed by a lift-off process in acetone. The remaining $\mathrm{Cr}$ mask pattern was transferred to the $\mathrm{Si}$ layer via reactive ion 
etching and subsequently removed with commercial etchant, providing the final sample. The SEM images of the fabricated sample are shown in Figs. 3(a) and 3(b), showing the dimers of the Si nanodisk of $652 \mathrm{~nm}$ diameter and $314 \mathrm{~nm}$ height. The aspect ratio of the Si nanodisk was lower than 1 so that the electric and magnetic dipoles are excited at closer wavelengths under the illumination of the TM polarized incidence, and the large spectral separation, which reduces the reflection of the metasurface, can be circumvented under the illumination of TE polarized incidence. The periodicities of the array were set to $1544 \mathrm{~nm}$ and $852 \mathrm{~nm}$ along and perpendicular to the dimer axis, respectively. These periodicities would meet the requirement of nanoresonator density, which needs to be high enough to avoid the strong diffraction effect. As shown in the SEM images, the array of identical Si dimers was fabricated uniformly throughout the metasurface.

We investigated the transmission and reflection of the fabricated Si dimer metasurface [Fig. 3(c)] using the FDTD method and FTIR spectroscopic technique. An FTIR microscope (Bruker Infinion) with $35 \times$ Cassegrain objectives was used to collect the transmission and reflection signals from the dielectric metasurfaces. The objective has a weightedaverage incident angle of approximately $25^{\circ}$. An aperture was used for all samples to limit the collection area to $50 \times 50 \mu \mathrm{m}^{2}$ so that dielectric metasurfaces fabricated by the EBL and RIE techniques can be observed. The transmission and reflection signals from the samples were normalized by the transmission in air and the reflection from an Au mirror

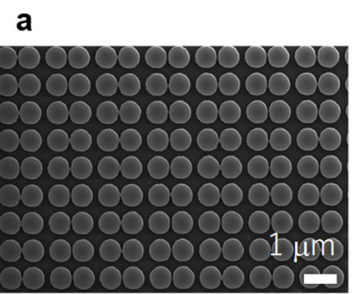

\section{b}

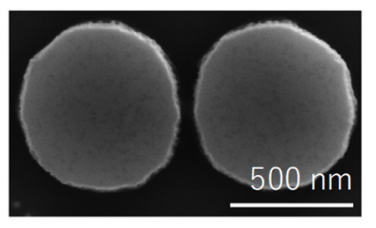

C
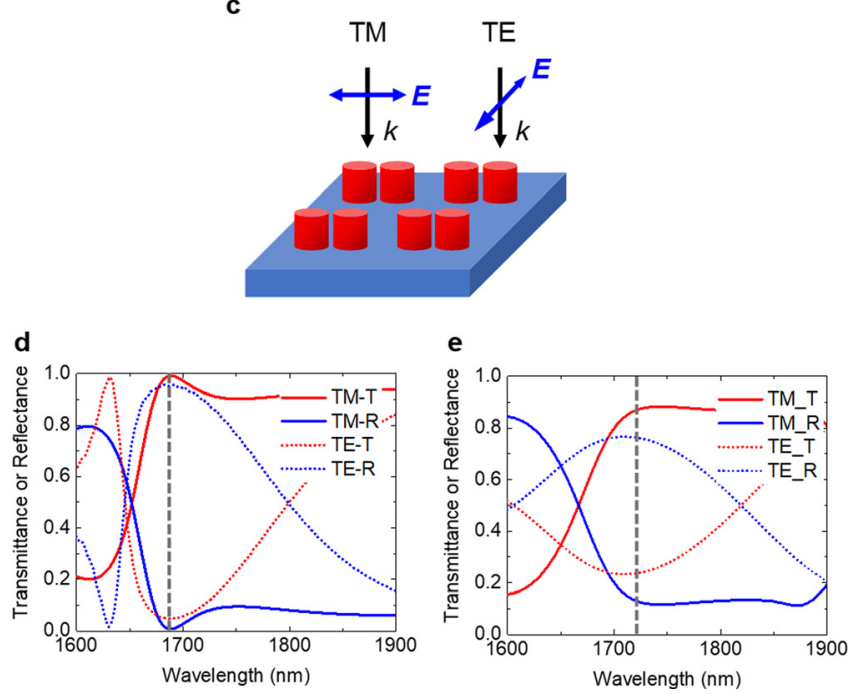

FIG. 3. (a) and (b) SEM images of the Si dimer metasurface fabricated on a sapphire substrate. (c) Schematic image of the Si dimer array on a sapphire substrate illuminated by the linearly polarized incident light. (d)-(e) Transmission (red lines) and reflection (blue lines) spectra of the Si dimer array under the illumination of TM (solid lines) and TE (broken lines) polarization in the FDTD simulations (d) and experiments (e). under the same settings, respectively. The transmission and reflection spectra are shown in Figs. 3(d)-3(e). In the FDTD simulation results, the metasurface exhibited the switch from the transmission of $99 \%$ to the reflection of $95 \%$ at $\lambda=1688 \mathrm{~nm}$ by changing the polarization from the TM to TE state. The experimental spectra also showed high transmission $(T=86 \%)$ and high reflection $(R=77 \%)$ at $\lambda=1718 \mathrm{~nm}$ depending on the incident polarization. These results theoretically and experimentally confirmed that the metasurface built of the dielectric nanodimers can switch from high transmission to high reflection by changing the linear polarization state of the incident light. The small disagreements between the numerical simulations and experiments might be attributed to the imperfection of the sample fabrication and also to the experimental measurement conditions which were different from the ideal simulation. We used Cassegrain objectives in the experiment to observe transmission and reflection of the dielectric metasurfaces in the NIR region, and therefore, the transmitted or reflected light normal to the substrate cannot be observed. This might cause the small disagreement with the simulation in the obtained optical responses. However, the experimental setup can still monitor a substantial amount of transmitted and reflected light and, hence, it does not change our conclusion. A very recent study revealed that arrays of large gold nanodisks with a rectangle lattice structure can tune polarizationdependent plasmonic lattice modes, which is in principle based on the diffraction of light. ${ }^{48}$ However, additional simulations of the array of dielectric dimers, which are not shown in this manuscript due to the lack of space, confirmed that the polarization switching in this study comes from the mode hybridizations of the excited electric and magnetic dipoles, rather than from the light diffraction.

In conclusion, we theoretically and experimentally demonstrated that metasurfaces built of arrays of high-index dielectric dimers can switch the optical response from high transmission to high reflection simply by changing the polarization of the incident light. Incident polarization can tune the hybridization modes of the excited electric and magnetic dipoles in the dielectric dimer. Numerical simulations of an array of Si spherical nanodimers revealed that the different mode hybridizations lead to the spectral overlap or separation between the electric and magnetic dipoles, depending on the incident polarization. These spectral features resulted in the linear polarization switching between high transmission and high reflection of the dielectric dimer metasurface. The switch between high transmission and reflection was experimentally confirmed using a Si disk nanodimer array fabricated on a sapphire substrate and an FTIR measurement setup. The tuneable control of the optical response of metasurfaces demonstrated in this letter could boost the development of a highly functional surface for flat optics.

The authors acknowledge funding provided by Grants from the EPSRC Reactive Plasmonics Programme EP/ MO13812/1, the Leverhulme Trust (UK), ONR Global, and the U.S. Army Transatlantic Research Office. S.A.M. further acknowledges the Lee-Lucas Chair. P.A. acknowledges Programa "Viera y Clavijo" de la Agencia Canaria de Investigación, Innovación y Sociedad de la Información 
(ACIISI) y la Universidad de las Palmas de Gran Canaria (ULPGC).

The authors declare no competing financial interest.

${ }^{1}$ N. Yu and F. Capasso, Nat. Mater. 13, 139-150 (2014).

${ }^{2}$ A. V. Kildishev, A. Boltasseva, and V. M. Shalaev, Science 339, 1232009 (2013).

${ }^{3}$ X. Ni, N. K. Emani, A. V. Kildishev, A. Boltasseva, and V. M. Shalaev, Science 335, 427 (2012).

${ }^{4}$ C. Pfeiffer, N. K. Emani, A. M. Shaltout, A. Boltasseva, V. M. Shalaev, and A. Grbic, Nano Lett. 14, 2491-2497 (2014).

${ }^{5}$ F. Aieta, P. Genevet, M. A. Kats, N. Yu, R. Blanchard, Z. Gaburro, and F. Capasso, Nano Lett. 12, 4932-4936 (2012).

${ }^{6}$ W. Li and J. Valentine, Nano Lett. 14, 3510-3514 (2014).

${ }^{7}$ S. Maier, Plasmonics: Fundamentals and Applications (Springer, New York, 2007).

${ }^{8}$ D. K. Gramotnev and S. I. Bozhevolnyi, Nat. Photonics 4, 83-91 (2010).

${ }^{9}$ J. B. Khurgin, Nat. Nanotechnol. 10, 2-6 (2015).

${ }^{10}$ D. M. Lin, P. Y. Fan, E. Hasman, and M. L. Brongersma, Science 345, 298-302 (2014).

${ }^{11}$ C. Wu, N. Arju, G. Kelp, J. A. Fan, J. Dominguez, E. Gonzales, E. Tutuc, I. Brener, and G. Shvets, Nat. Commun. 5, 3892 (2014).

${ }^{12}$ Y. Yang, I. I. Kravchenko, D. P. Briggs, and J. Valentine, Nat. Commun. 5, 5753 (2014).

${ }^{13}$ A. Arbabi, Y. Horie, M. Bagheri, and A. Faraon, Nat. Nanotechnol. 10, 937-943 (2015).

${ }^{14}$ S. Kruk, B. Hopkins, I. Kravchenko, A. Miroshnichenko, D. N. Neshev, and Y. S. Kivshar, APL Photonics 1, 30801 (2016).

${ }^{15}$ R. Paniagua-Domínguez, Y. F. Yu, A. E. Miroshnichenko, L. A. Krivitsky, Y. H. Fu, V. Valuckas, L. Gonzaga, Y. T. Toh, A. Y. S. Kay, B. Luk'yanchuk, and A. I. Kuznetsov, Nat. Commun. 7, 10362 (2016).

${ }^{16}$ N. Odebo Länk, R. Verre, P. Johansson, and M. Käll, Nano Lett. 17, 3054-3060 (2017).

${ }^{17}$ M. M. Sigalas, D. A. Fattal, R. S. Williams, S. Y. Wang, and R. G. Beausoleil, Opt. Express 15, 14711-14716 (2007).

${ }^{18}$ A. B. Evlyukhin, C. Reinhardt, A. Seidel, B. S. Luk'yanchuk, and B. N. Chichkov, Phys. Rev. B 82, 45404 (2010).

${ }^{19}$ A. Garcia-Etxarri, R. Gomez-Medina, L. S. Froufe-Perez, C. Lopez, L. Chantada, F. Scheffold, J. Aizpurua, M. Nieto-Vesperinas, and J. Saenz, J. Opt. Express 19, 4815-4826 (2011).

${ }^{20}$ A. I. Kuznetsov, A. E. Miroshnichenko, Y. H. Fu, J. B. Zhang, and B. Luk'yanchuk, Sci. Rep. 2, 492 (2012).

${ }^{21}$ A. B. Evlyukhin, S. M. Novikov, U. Zywietz, R. L. Eriksen, C. Reinhardt, S. I. Bozhevolnyi, and B. N. Chichkov, Nano Lett. 12, 3749-3755 (2012).

${ }^{22} \mathrm{P}$. Albella, R. A. de la Osa, F. Moreno, and S. A. Maier, ACS Photonics 1, 524-529 (2014).

${ }^{23}$ Q. Zhao, J. Zhou, F. Zhang, and D. Lippens, Mater. Today 12, 60-69 (2009).

${ }^{24}$ I. Staude, A. E. Miroshnichenko, M. Decker, N. T. Fofang, S. Liu, E. Gonzales, J. Dominguez, T. S. Luk, D. N. Neshev, I. Brener, and Y. Kivshar, ACS Nano 7, 7824-7832 (2013).
${ }^{25}$ M. Decker, I. Staude, M. Falkner, J. Dominguez, D. N. Neshev, I. Brener, T. Pertsch, and Y. S. Kivshar, Adv. Opt. Mater. 3, 813-820 (2015).

${ }^{26}$ K. E. Chong, I. Staude, A. James, J. Dominguez, S. Liu, S. Campione, G. S. Subramania, T. S. Luk, M. Decker, D. N. Neshev, I. Brener, and Y. S. Kivshar, Nano Lett. 15, 5369-5374 (2015).

${ }^{27}$ K. E. Chong, L. Wang, I. Staude, A. R. James, J. Dominguez, S. Liu, G. S. Subramania, M. Decker, D. N. Neshev, I. Brener, and Y. S. Kivshar, ACS Photonics 3, 514-519 (2016).

${ }^{28}$ B. Slovick, Z. G. Yu, M. Berding, and S. Krishnamurthy, Phys. Rev. B: Condens. Matter Mater. Phys. 88, 165116 (2013).

${ }^{29}$ P. Moitra, B. A. Slovick, Z. G. Yu, S. Krishnamurthy, and J. Valentine, Appl. Phys. Lett. 104, 171102 (2014).

${ }^{30}$ P. Moitra, B. A. Slovick, W. Li, I. I. Kravchencko, D. P. Briggs, S. Krishnamurthy, and J. Valentine, ACS Photonics 2, 692-698 (2015).

${ }^{31}$ Z. Ma, S. M. Hanham, P. Albella, B. Ng, H. T. Lu, Y. Gong, S. A. Maier, and M. Hong, ACS Photonics 3, 1010-1018 (2016).

${ }^{32}$ J. Sautter, I. Staude, M. Decker, E. Rusak, D. N. Neshev, I. Brener, and Y. S. Kivshar, ACS Nano 9, 4308-4315 (2015).

${ }^{33}$ A. Komar, Z. Fang, J. Bohn, J. Sautter, M. Decker, A. Miroshnichenko, T. Pertsch, I. Brener, Y. S. Kivshar, I. Staude, and D. N. Neshev, Appl. Phys. Lett. 110, 071109 (2017).

${ }^{34}$ J. Hu, X. Zhao, Y. Lin, A. Zhu, X. Zhu, P. Guo, B. Cao, and C. Wang, Sci. Rep. 7, 41893 (2017).

${ }^{35}$ J. Zhao, Y. Chen, and Y. Feng, Appl. Phys. Lett. 92, 071114 (2008).

${ }^{36}$ P. Albella, M. A. Poyli, M. K. Schmidt, S. A. Maier, F. Moreno, J. J. Saenz, and J. Aizpurua, J. Phys. Chem. C 117, 13573-13584 (2013).

${ }^{37}$ R. M. Bakker, D. Permyakov, Y. F. Yu, D. Markovich, R. PaniaguaDominguez, L. Gonzaga, A. Samusev, Y. Kivshar, B. Luk'yanchuk, and A. I. Kuznetsov, Nano Lett. 15, 2137-2142 (2015).

${ }^{38}$ U. Zywietz, M. K. Schmidt, A. B. Evlyukhin, C. Reinhardt, J. Aizpurua, and B. N. Chichkov, ACS Photonics 2, 913-920 (2015).

${ }^{39}$ L. Lewin, J. Inst. Electr. Eng. 94, 65-68 (1947).

${ }^{40}$ J. M. Geffrin, B. Garcia-Camara, R. Gomez-Medina, P. Albella, L. S. Froufe-Perez, C. Eyraud, A. Litman, R. Vaillon, F. Gonzalez, M. NietoVesperinas, J. J. Saenz, and F. Moreno, Nat. Commun. 3, 1171 (2012).

${ }^{41}$ Y. H. Fu, A. I. Kuznetsov, A. E. Miroshnichenko, Y. F. Yu, and B. Luk'yanchuk, Nat. Commun. 4, 1527 (2013).

${ }^{42}$ S. Person, M. Jain, Z. Lapin, J. J. Saenz, G. Wicks, and L. Novotny, Nano Lett. 13, 1806-1809 (2013).

${ }^{43}$ X. Chen, T. M. Grzegorczyk, B. I. Wu, J. Pacheco, and J. A. Kong, Phys. Rev. E 70, 016608 (2004).

${ }^{44}$ D. R. Smith, D. C. Vier, T. Koschny, and C. M. Soukoulis, Phys. Rev. E. 71, 036617 (2005).

${ }^{45}$ E. Prodan, C. Radloff, N. J. Halas, and P. Nordlander, Science 302, 419-422 (2003).

${ }^{46}$ P. Nordlander, C. Oubre, E. Prodan, K. Li, and M. I. Stockman, Nano Lett. 4, 899-903 (2004).

${ }^{47}$ V. Giannini, A. I. Fernández-Domínguez, Y. Sonnefraud, T. Roschuk, R. Fernández-García, and S. A. Maier, Small 6, 2498-2507 (2010).

${ }^{48}$ R. R. Gutha, S. M. Sadeghi, and W. J. Wing, Appl. Phys. Lett. 110, 153103 (2017). 